\title{
A Traceable and Reliable Electronic Supply Chain System Based on Blockchain Technology
}

\author{
Shaniar Tahir Mohammed*, Jamal Ali Hussien \\ Department of Computer, College of Science, University of Sulaimani, Sulaymaniyah, Iraq
}

\section{A B S T R A C T}

Electronic supply chain (ESC) is a network among the parties of a supply chain system, such as manufacturers, suppliers, and retailers. It recodes all the processes involved in the distribution of specific products until transported to final customers. Blockchain (BC) technology is a decentralized network that records all the transactions in real-time and is used in many areas such as cryptocurrency. In this paper, we work on an ESC system that records all the transactions based on BC technology using a drug supply chain system as a case study. The recording of the transactions consists of three main stages. First, all the parties of the ESC system are represented in the BC network as clients with unique identities. Second, all the information related to a specific drug is recorded inside the transaction and each transaction has its own signature. Finally, all the transactions of the drug from the manufacture to the patient are recorded inside a block with a unique identity for each block. These steps inside the BC are performed based on security cryptography mechanisms, such as rivest-shamir-adleman (RSA) and secure hash algorithm SHA. The results illustrate that the proposed approach protects the drugs from counterfeiting, ensures the reliability, and provides a real-time tracking system for the transactions that have occurred among ESC parties.

Index Terms: Blockchain, Block, Electronic Supply Chain, Monitoring, Reliability, Traceability

\section{INTRODUCTION}

Supply chains can be defined as a series of interconnected activities between manufacturers and customers, which include the coordination, planning, and controlling of products and services [1]. The classical supply chain has many issues such as need for paperwork; speed limits; and lack of traceability, security, and reliability [2]. The ESC records all the activities and transactions of supply chains electronically [1], [2], [3]. In the logistics process in an ESC, the products can be transported in a way that the parties (manufacture, supplier, distributor, wholesaler, retailer,

\section{Access this article online}

DOI: 10.21928/uhdjst.v4n2y2020.pp132-140

E-ISSN: $2521-4217$

P-ISSN: 2521-4209

Copyright $@ 2020$ Shaniar. This is an open access article distributed under the Creative Commons Attribution Non-Commercial No Derivatives License 4.0 (CC BY-NC-ND 4.0) and customer) of the network can ensure the quality of goods [1], [4], [5].

The blockchain (BC) is a decentralized peer-to-peer ( $\mathrm{p} 2 \mathrm{p}$ ) network distributed ledger, with unlimited digital transactions across the network without the need of third-parties. This technology can be used in different areas such as cryptocurrency, since it has a useful number of features such as reliability and traceability [6], [7], [8]. BC has different types depending on the organization or the company that uses it or on the architecture of the network [6]. In BC technology, every single node or client has a copy of the ledger in the network after a consensus from all participants [2], [6]. BC has four basic features: Decentralization, openness, security, and privacy [2], [4]. BC and distributed databases (DBs) are different in their structures. In addition, in $\mathrm{BC}$ systems the transactions are controlled and managed by all the participants while in database system DBs the transactions are managed by a single entity [6].

Corresponding author's e-mail: Shaniar Tahir Mohammed, Department of Computer, College of Science, University of Sulaimani, Sulaymaniyah, Iraq. E-mail: shaniar.mohammed@univsul.edu.iq 
Many researchers have worked on the process of merging the electronic supply chain (ESC) with BC using different approaches. However, either the researches stay theoretical and have not been implemented [4] or it does not fully benefit from all the security features of $\mathrm{BC}$ for improving reliability, traceability, and the monitoring process of the ESC systems [9].

In this paper, $\mathrm{BC}$ technology is used to create a reliable architecture for the ESC process and solves the problem of supply chain systems, such as reliability and traceability. Regarding the drug safety issues, the aim of this research was to help authorities in Iraqi Kurdistan to monitor and trace drug transportation from the manufacturers all the way to the patients in a secure and reliable way. In particular, this model is helpful in reducing paperwork, monitoring drug flow between ESC clients, protecting the drug from counterfeiting, tracing the transactions, reducing cost, and protecting the information flow between clients.

The proposed model is based on three main parts of $\mathrm{BC}$ technology used in ESC architecture: Clients, Transactions, and Blocks. The Clients represent the ESC parties, such as suppliers and manufacturers. Each client has a unique identity based on the security mechanism RSA-1024. The Transactions hold the drug flow information in ESC between all parties. The proposed model generates a signature for each transaction based on RSA-1024 algorithm, for protecting the ESC from unknown transactions and also from modification. The Blocks hold the transaction between the ESC parties, each block holds five transactions from the manufacturer to the final destination, the patient. Each block has a unique identity based on the SHA-256 algorithm. The information flow of drug ESC model is decentralized and shared between all the clients and recorded in real-time enabling tracking and monitoring of the activities by all the participants.

\subsection{Problem Statement}

The classical supply chain has some problems such as depending on paperwork to exchange information; high cost; and lack of reliability, traceability, and trust. While in the ESC, all the information and processes are captured and recorded in an electronic way, which is helpful in eliminating paperwork, reducing cost, and increasing reliability, since all processes can be monitored by all parties of the ESC system.

$\mathrm{BC}$ is a helpful technology that can be used with an ESC to record all the information in a decentralized way. Then, the processes of the ECS and can be monitored by all participants of the network without needing a third party.
In addition, it increases reliability because in $\mathrm{BC}$ various security mechanisms are used when recording supply chain processes. Furthermore, traceability can be improved based on $\mathrm{BC}$ technology, since the encryption of the information prevents unknown transactions and unknown clients protecting the data from modification. All the transactions that have occurred in the ESC can be monitored in real-time.

In Iraqi Kurdistan, there does not exist an electronic system for recording and monitoring drug information and the transactions between the parties of the supply chain system. Therefore, it is difficult to establish reliability and provides an efficient traceability of drugs. For our case study, we work on the pharmaceutical system for drug transportation from manufacturers to final destination patients in Iraqi Kurdistan using ESC with BC technology.

The proposed system allows us to protect the drug from counterfeiting, enables traceability of drugs, and increase reliability between all parties in the ESC system. The information flow between all parties is held in BC in a secure way without a third-party involvement; therefore, the authorities can easily monitor drug transportation and transactions.

\section{BACKGROUND}

\subsection{The Structure of ESC Systems}

The ESC is a network between the producer of the specific product and the supplier to record the information about all the processes of product transportation and improving health and safety, speed, cost, scalability, and transparency while distributing to consumers [10], [11], [12]. In this network, there exist various entities, such as people, resources, and information that need to be recorded in a fast and secure way. The general structure of the ESC includes the following participants:

a) Provider: It provides raw materials to the suppliers, such as the raw materials used in producing drugs, food ingredients, and automobile parts [13].

b) Supplier: It is somebody who is responsible for the actions of producing the raw materials, like a farmer.

c) Manufacturer: The manufacturer performs various actions to produce specific products.

d) Distributor: The distributor is responsible for moving the product to the retailers.

e) Retailer: It is responsible for marketing the goods, such as local stores, supermarkets, pharmacies, or car shops [3], [14]. 
f) Consumer: The consumer buys a product and checked it to ensure originality. For instance, a patient who buys the drug in a pharmacy [13].

Fig. 1 illustrates all the stages of the general ESC process:

\subsection{BC Architecture}

BC technology was conceptualized for the first time by Satoshi Nakamoto in 2008 and used in various areas especially for cryptocurrencies like "Bitcoin" [15], [16], [17]. This technology is a decentralized p2p mesh network of nodes linked to each other and contains blocks without the need to be managed by a third-party. Several layers govern BC operations and generate the protocols for $\mathrm{BC}$ applications.

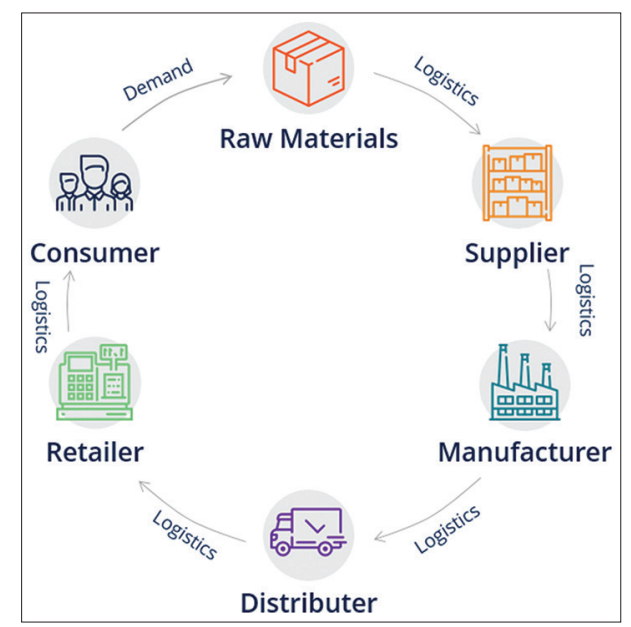

Fig. 1. General structure of the electronic supply chain.
$\mathrm{BC}$ generally has two main types: Public $\mathrm{BC}$ and private BC. Public BC is decentralized where parties can access the current and previous records. While private $\mathrm{BC}$ is a centralized network that is privately available for organizations that have a limited number of participants. Private $\mathrm{BC}$ is less secure than public BC. Fig. 2 shows an overall structure of the BC architecture [18]:

The $\mathrm{BC}$ contains a sequence of techniques that are used to recording transactions in real-time between the parties of the network (sender and recipient). The information is held inside blocks and each block links to a previous block, as shown in Fig. 2. The BC provides security features such as cryptographic hash, digital signature, and distributed consensus mechanism [13], [18], [19].

Fig. 3 illustrates the general architecture of $\mathrm{BC}$ in the Bitcoin process [15]. Each transaction that occurs in a BC encapsulates the phases is shown in Fig. 3. In the case of supply chain systems, all logistic processes from producing a specific good until delivering it to customers are recorded.

The Bitcoin cryptocurrency process consists of the following six stages. The same stages are used for our proposed system.

1. The Transaction: The transaction holds information, for example, in an ESC transaction; it includes the sender and receipt identity, date and time, the quantity of the goods, name of the product, location, and so on.

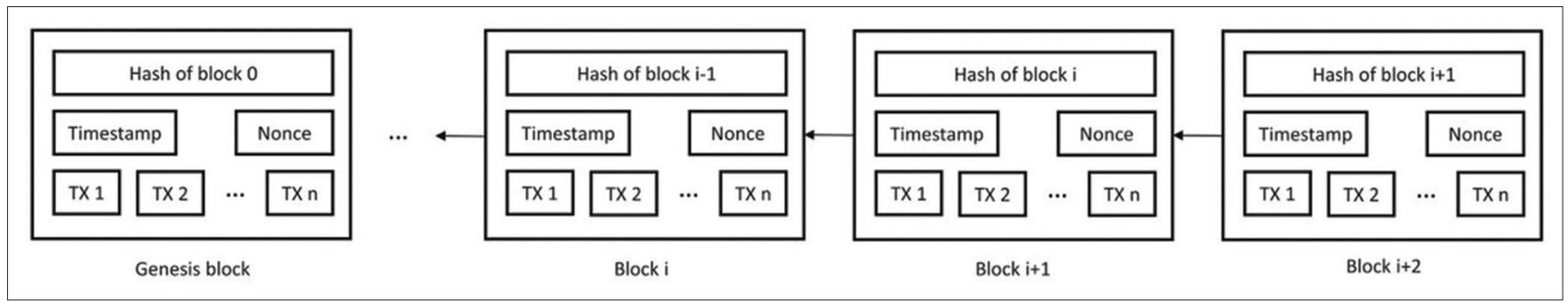

Fig. 2. Block structure in the blockchain technology.

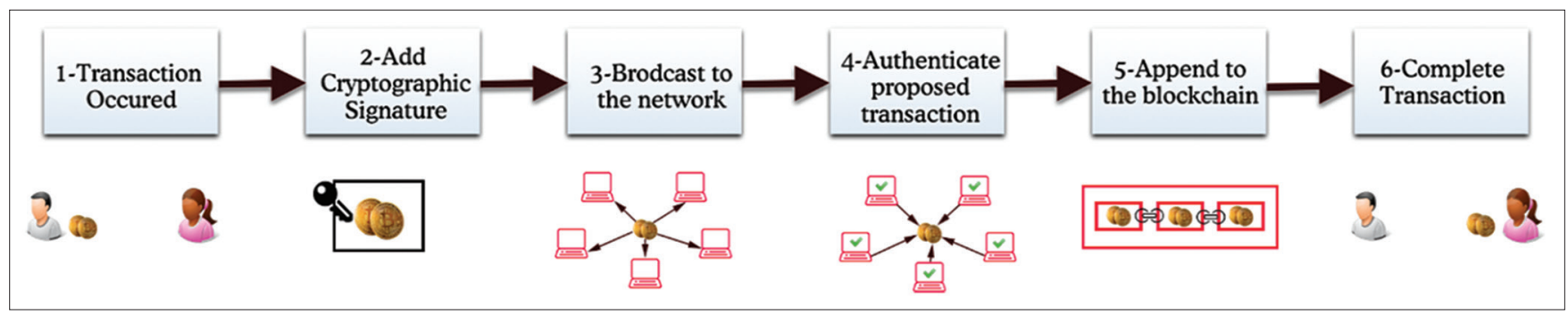

Fig. 3. Cryptocurrency with blockchain architecture. 
2. A cryptographic signature is the second stage of the $B C$ architecture to provide a secure algorithm function to implement a cryptographic signature to both sender and receipt identity using cryptographic algorithms, such as SHA256, SHA512, and RSA.

3. Broadcasting the Transaction: In this stage, the transaction must be broadcasted to the network for the authentication process.

4. The transaction verification: The transaction must be authenticated and verified by all the parties inside the decentralized network.

5. Digital ledger: After notifying all the parties of the BC of the new transactions, they are added to the digital ledger and appended to the BC.

6. Transaction completion: Finally, the transaction is completed and the money or the information related to it is transferred to the receipt and added to the block. Each block is linked to the previous one.

\subsection{Cryptographic Algorithms}

The secure hash algorithm SHA is one of the cryptographic hash functions designed to hold data securely by transforming the data into hash code using its own family of types such as SHA1, SHA224, SHA256, and SHA512. Each of them has a different size in bytes [20].

The RSA cryptographic system is used for data encryption and decryption with two keys (private key and public key), when the private key is only known to the owner and the public key is publicly available and can be accessed by everyone [21], [22]. The size of the key of RSA should be 1024 bits or higher. The public key is used for encryption while the private key is used for decryption [21].

\section{THE RUNNING EXAMPLE}

We describe the supply chain process in an earlier section. We show a small example of a supply chain process between two clients known as the manufacturer and the supplier for transporting a drug between them. Table 1 shows the

TABLE 1: A simple transaction information
occurred between two clients in a supply chain
system

information related to one transaction occurred between two clients in the supply chain. This transaction is taken from a large set of transactions from the pharmaceutical system in the Iraqi Kurdistan. We use the information about a specific drug to demonstrate various transactions in our proposed ESC with BC. In the upcoming sections, we show more transactions about a specific drug. The structure of the transactions contains all the information needed for the drug supply chain process, such as drug $i d$, drug name, sender_id, recipient_id, location, size, and date and time.

In ESC systems, the information can be stored electronically with a centralized system without real-time recording of the process, which is not secure. However, public BC systems with ESC are secure since they are decentralized and there is no need for third-parties to manage the process. Public BC systems are reliable and transactions can be traced at each stage of the ESC in real-time.

\section{RELATED WORK}

Randhit kumar and Rakish Tripathi [9] proposed a traceability structure for supply chain based on BC technology to medicine system or drug manufacturing to protect the drug while transported from the manufacturer to the consumer, they used $\mathrm{BC}$ to encrypt the QR code of the drugs to protect the drug from counterfeiting. The methodology of this research provides a structure to protect the medicine supply chain from a man-in-the-middle attack.

$\mathrm{BC}$ in various case studies and different sectors of industry such as the pharmaceutical supply chain is used by $S$. Aich, S. Chakraborty, M. Sain, H. Lee, and H. Kim [13] to ensure the tractability of drugs and to improve the efficiency of supply chain network. They mentioned the problems occurred in different sectors of the conventional supply chain and find solutions. They compare the traditional supply chain to a digital supply chain based on BC and Internet of Things (IoT) for pharmaceutical process to track and protect drugs from counterfeiting. IoT was used to record digital identification for all products to provide trustworthiness in the digital supply chain system. All the participants of the system in the network can ensure the transparency of the information recorded in the $\mathrm{BC}$.

M. P. Caro, M. S. Ali, M. Vecchio, and R. Giaffreda [23] proposed a new agriculture food supply chain based $\mathrm{BC}$ architecture with IoT to keep the system from data tampering. The integrity of the data and traceability of the process is 
conducted using various $\mathrm{BC}$ mechanisms such as ethereum and hyperledger sawtooth.

A three-tier architecture based on BC was proposed by S. Malik [10] to ensure data availability to consumers and to provide scalability for handling transaction loads while keeping the history and confidential information safe when delivering to other parties of a supply chain process.

\section{BC -BASED ESC SYSTEM}

In this section, we propose an ESC model, which enables traceability, reliability, monitoring of drug transactions in the Iraqi Kurdistan based on the BC technology. We worked on the pharmaceutical supply chain by recording logistic steps of the ESC to transport a drug from the manufacturer to a patient using BC technologies. Fig. 4 shows a block diagram of the proposed model.

In our proposed system, transactions occur among supply chain parties in pharmaceutical process according to these instructions:

1. Capturing the transaction $n$ in the pharmaceutical supply chain system between client A and client B.
2. Based on the RSA-1024 algorithm, generating a unique identity for each one of the five different types of clients. The identities are shared between all the participants of the BC. Table 2 shows the client identities.

3. Build a unique transaction signature using RSA-1024, which facilitates traceability and the identification of the drug transported between the clients.

4. Each transaction holds the sender and recipientidentities, item information, date and time, and other related information.

5. Typically, each drug requires five transactions between the clients to be delivered from the manufacturer to the patient in the supply chain process. These transactions are added to a single block $m$.

\section{TABLE 2: Client identity while applied RSA-1024 algorithm in ESC}

\begin{tabular}{llc}
\hline Client Type & Client Name & Client Identity \\
\hline Manufacturer & Sanofi & 30819f300d06092a864886f70d \\
& & $01010105000381 \ldots \ldots$. \\
Supplier & Awafi Medicine & 06c48043be02d767f023a62ff5 \\
& Store & b7ec7c0bc08e9bd...... \\
Wholesaler & KMCA & c873e055bf3971a944882e78 \\
& & eb82ed0045af93c2....... \\
Retailer & Shar & f3a6765fb8755bfc8543ef2d691 \\
& & d3799ec281cda96....... \\
Patient & Ahmed & 0dfb14bfb66ada3c097353d2cc \\
& & $58608 \mathrm{e} 8659 \mathrm{cbbe} . \ldots \ldots$ \\
\hline
\end{tabular}

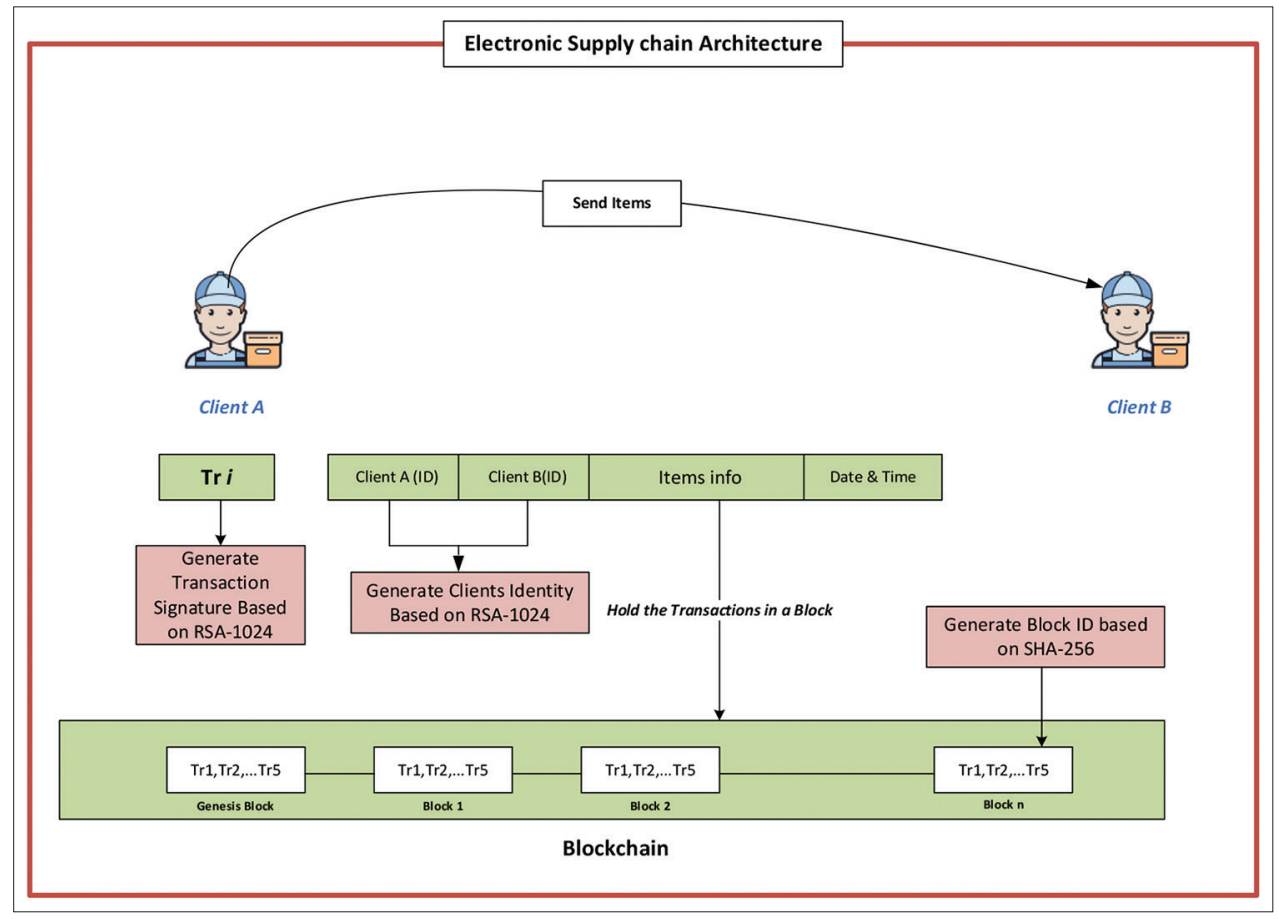

Fig. 4. Block diagram of the electronic supply chain process using blockchain technology. 
6. Before adding them to the $\mathrm{BC}$, a unique identity based on the $S H A-256$ algorithm is generated for each block.

7. The block $m$ added to the BC and each block linked to the previous one. This makes blocks to be easily traceable and reliable, and facilitate real-time monitoring.

In Fig. 5, the main parts of the proposed ESC system are illustrated. The system records any transaction that has occurred inside the ESC using BC. The ESC includes five types of clients: Manufacturer, supplier, wholesaler, retailer, and patient. Drugs are produced by the manufacturer, transported to the supplier, wholesaler, and then to the warehouse or the retailer. Finally, the drugs will be delivered to the patient. All these transactions can be tracked and recorded securely without need to third-party's involvement.

\subsection{Transaction Processes}

Each transaction has its own signature; they are created with RSA-1024 algorithm. With these signatures, we can protect our ESC transactions from any unknown transactions. Fig. 6 depicts a small transaction between two clients in the ESC.

We hold five transactions inside each block of the BC, one transaction between each two clients starting from the manufacturer and ending with the patient. Table 3 shows three transaction signatures as an example.

\subsection{The Block Structure of Our BC System}

In this research, another main part is the generation of a unique block for the ESC's transactions before adding it to the $\mathrm{BC}$. Block identities are generated with the SHA-256 algorithm.
In general, inside the $\mathrm{BC}$, we have an unlimited number of blocks, which means that the system can generate any number of blocks, but in the proposed approach worked on 2000 blocks, each block holds five transactions in the pharmaceutical supply chain process in Iraqi Kurdistan. The size of each block is between 830 and 890 bytes depending on the amount of information saved inside each transaction and held in a block, the BC is not reseted after holding the transaction, and the transactions history remain inside the blocks. Each block in the BC system is protected from modification because these blocks are shared in a decentralized network and each block is linked to the previous block with its own identity. Only the participants of the supply chain system can see the information inside the blocks, which enables the clients to easily monitor the processes of ESC. The average time duration between the current block and the previous block is $0.0056 \mathrm{~ms}$. Table 4 shows four blocks with current and previous identities.

\section{RESULTS AND DISCUSSION}

In the proposed system, we can ensure drug quality and the correctness of information related to the drug manufacturing process. Our system creates a secure channel among parties based on $\mathrm{BC}$ technology, which provides traceability of drugs, reduces cost, enhances reliability, eliminates paperwork, and facilitates monitoring by all clients in the pharmaceutical process.

Table 5 contains a chain of five transactions inside a single block (\#block112) regarding the transportation of a specific drug from the manufacturer all the way to the patient. The

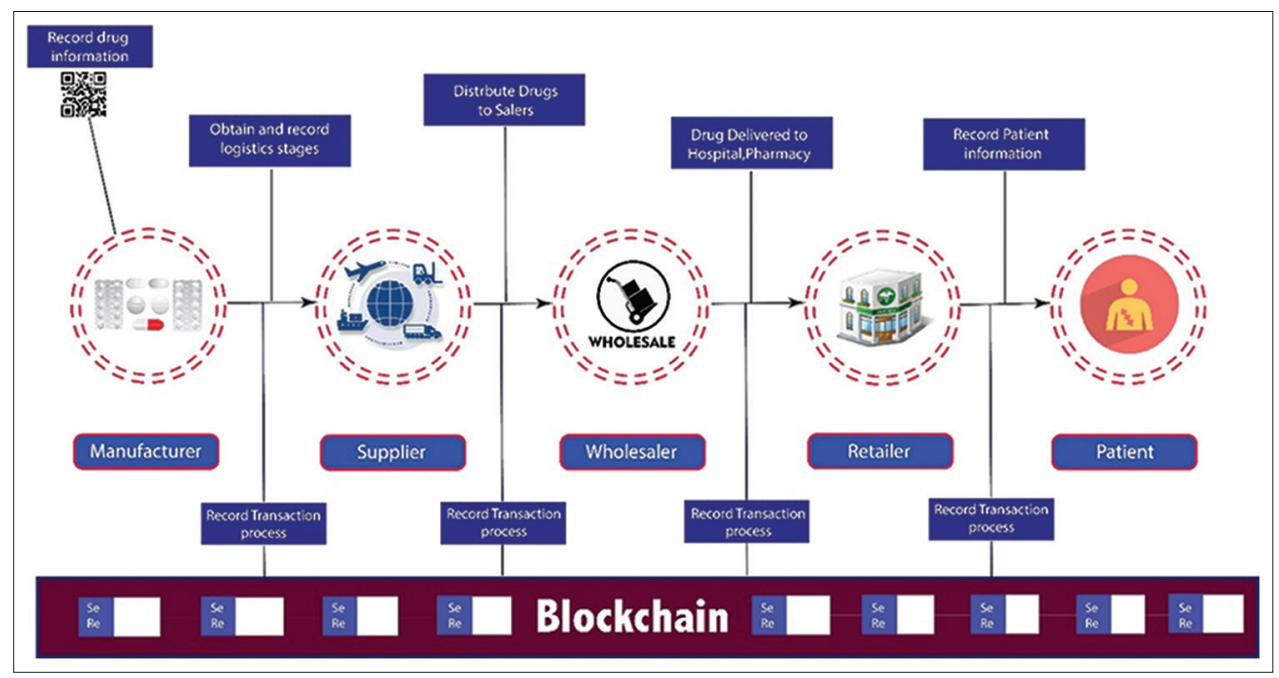

Fig. 5. Pharmaceutical supply chain process in ESC. 


\section{TABLE 3: Three transaction signature while applied RSA-1024 algorithm in ESC}

1bee9567d03e61c48e748cb2983ee4bfd308009555e45d6fe9bbbed051e338055e9950a664e280048268b8457a16ce22a8b699b3e11e0f8d553e 23541b328e949f18c7db20cd44a9c60153d3dff3538984aae8eb1fa117d54fe84e2cfd261a659cc05c97e87e7b7078c101d81595ebcf4610f ca0172799935f82df0125b04e82

6f606e75128216b1562ca131ffdc6bae4d9967636244cac72478ea5e8764bf86824f6ad9ed250b7c031896604e969dd3cceb5989a556bf31 e0a39aca8d84d69e41740221592f4b0c0feacb34f0068c5ef5d731108cbe8e6c36503d1d158332eeb6b6cd62b734dd147409d11d085eb65 dccb4d0874c384ddbf2b0495aec858c8c

8b3c55fbe861e80937fb1ac70fa7781c041bd7af30249b0e42f7f6553e265a260a87d00d44a59942a34f3cbf168a7a54a1afb9dc40e946ff43d0 d6a7e69d72eea8c96b8da2306e7e334597cc7192e5f040318bfeea61af225c2b0da26b68b6195ef4066c6b8a9f65c9d49607bf68b340359ae bb8e464de7d1b6c87f687851bb70

\begin{tabular}{|c|c|c|}
\hline Block number & $\begin{array}{c}\text { Block current } \\
\text { identity (SHA-256) }\end{array}$ & $\begin{array}{c}\text { Block previous } \\
\text { identity (SHA-256) }\end{array}$ \\
\hline \#Block112 & $\begin{array}{c}\text { 6ad6d74005e15af42 } \\
\text { 18b693b3c...... }\end{array}$ & $\begin{array}{c}\text { 0a6b88cb1c75ab } \\
\text { 970fd11e4.............. }\end{array}$ \\
\hline \#Block251 & $\begin{array}{c}\text { 23075fc5076b836 } \\
\text { 136a3a92e9...... }\end{array}$ & $\begin{array}{c}\text { a9f893431c9c961 } \\
\text { bfb7896483...... }\end{array}$ \\
\hline \#Block512 & $\begin{array}{l}97 \mathrm{fb} 8026 \mathrm{~b} 07 \mathrm{ed} 4 \mathrm{f} 1 \\
\mathrm{f} 73 \mathrm{~d} 23 \mathrm{f} 31 \ldots \ldots\end{array}$ & $\begin{array}{c}\text { 1ca161f2094af3f6e } \\
\text { fba34h11...... }\end{array}$ \\
\hline \#Block600 & $\begin{array}{c}906886 \mathrm{c} 8477237 \mathrm{~b} \\
80 \mathrm{~b} 0 \mathrm{de} 1 \mathrm{fb} 2 \ldots \ldots\end{array}$ & $\begin{array}{c}\text { aa45b1772c74e330 } \\
74 d 0 c 061 a . . . . .\end{array}$ \\
\hline
\end{tabular}

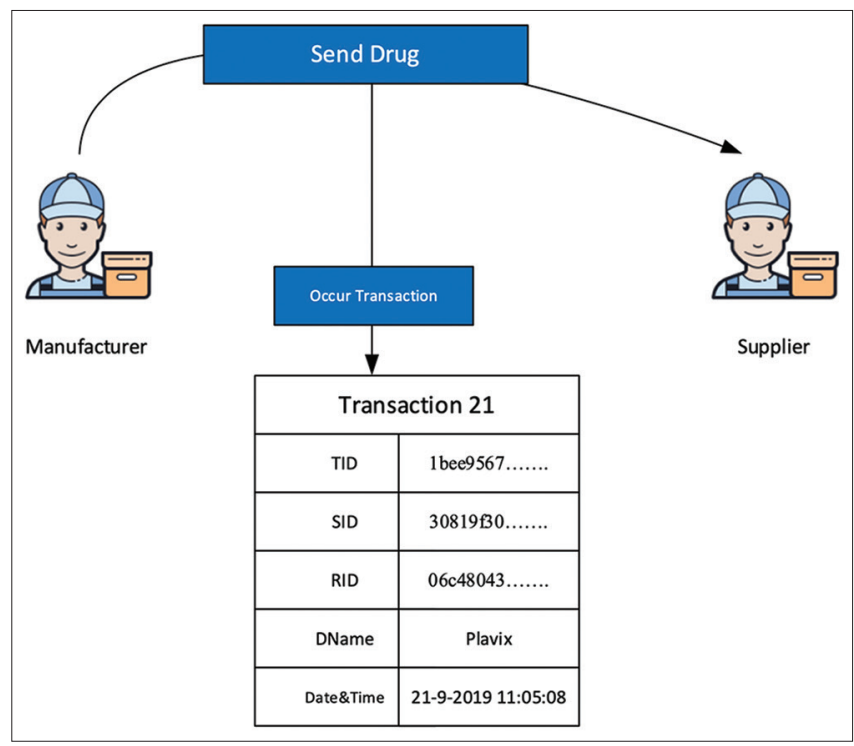

Fig. 6. Transaction structure and signature in ESC.

drug has its own unique identifier. There are unique identifiers for each of the sender and the receiver per transaction along with the date and time of the transaction.

In Table 6, we choose a transaction (\#transaction21) among the five transactions of the block (\#block112) between the supplier, $\mathrm{R} A S A N$, and the wholesaler, $K M C A$, showing all the information of that transaction. The detail about the transaction includes the location, date, and time, and size.

\subsection{Traceability}

In our proposed system, each block contains the transactions that are related to one single drug. Each drug has a unique identifier, for example, \#block 112 is encrypted with the SHA256 cryptography algorithm and linked to the previous block (Table 6). In this way, we can trace the transaction between these two clients. We can determine when the transaction has started (DateTime), which clients participate in the transaction (Sender and Recipient IDs), where is the current location of the drug (Supplier and Wholesaler locations), and whether the transaction has occurred or it is in a waiting state.

The information is recorded in real-time, which means we can trace each drug transported between the clients in real-time. The transactions can be monitored by all the participants, without modification by an unknown client or user. Since the network is decentralized, we do not need a third-party for managing the information of the transactions that are held inside the blocks.

\subsection{Reliability and Monitoring}

The reliability of the information is another feature of the proposed approach. Since our system uses several security mechanisms with BC technology, such as RSA-1024 and SHA-256 algorithms. These algorithms are used for generating a unique identity for each participant (sender identity and recipient identity), transaction (transaction identity), and block (block identity) of the ESC system, as shown in Table 5. With these identities, we can trust the transactions. $\mathrm{BC}$ has an important process called consensus algorithm, which provides agreements among clients of the $\mathrm{BC}$ network about the data. Based on the consensus algorithm a participant can generate new blocks that must be accepted by the other parties of the system. This is useful for improving the reliability and ensuring the traceability of the system.

There is an important feature inside the transactions, which is the transaction type. Its value is either verified or unverified. If a client notices that some identities have been changed or the 
TABLE 5: Simple drug information for five transactions inside one block in the blockchain system

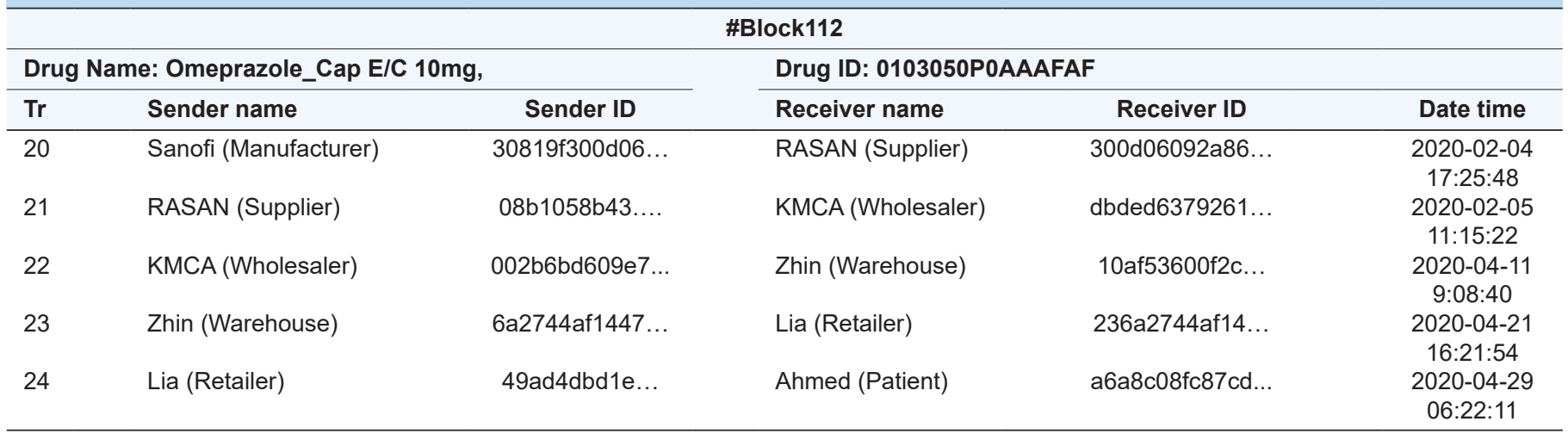

\begin{tabular}{|c|c|}
\hline \multicolumn{2}{|l|}{ \#Block112 } \\
\hline \multicolumn{2}{|l|}{ \#Transaction21 } \\
\hline Transaction ID & $\begin{array}{c}\text { 1bee9567d03e61c48e748cb2983ee4bfd } \\
\text { 308009555e45d6f........... }\end{array}$ \\
\hline Sender ID & $08 b 1058 b 43 \ldots \ldots \ldots \ldots$ \\
\hline Recipient ID & 300d06092a86........ \\
\hline Supplier Location & Iraq \\
\hline Wholesaler Location & Iraq \\
\hline DateTime & $10-1-2020$ 08:22:12 \\
\hline Drug ID & 0103050POAAAFAF \\
\hline Drug Name & Omeprazole_Cap E/C 10mg \\
\hline Size in Block & $15 \overline{0}$ Byte \\
\hline Block_Id & $\begin{array}{c}\text { 6ad6d74005e15af4218b693b3cb1fb79538 } \\
\text { 159d5db568f7528c...... }\end{array}$ \\
\hline Block_Prev_ld & $\begin{array}{c}\text { 0a6b88cb1c75ab970fd11e49f1db8d237d9 } \\
\text { bf819ef1165fdb0fb...... }\end{array}$ \\
\hline Transaction Type & Verified \\
\hline Situation & Occurred \\
\hline
\end{tabular}

drug information has been modified, then the value of the field transaction type is changed from verified to unverified and all the participants of the network are notified about this modification. This prevents the transaction to be faked and protects the drug from counterfeiting. This process enables the reliability of the ESC system and establishes trust among the participants of the network. Since all the transactions happen in real-time, all participants as well as the authorities can monitor the process of drug transportation between all the clients of the ESC system.

\section{CONCLUSIONS AND FUTURE WORK}

In this research work, $\mathrm{BC}$ technology is used for recording information flow between supply chain parties using the pharmaceutical supply chain system in Iraqi Kurdistan as a running example. The proposed system generates unique identifiers for the clients and the transactions regarding a specific drug and stores them along with the drug information inside a block of the $\mathrm{BC}$ system. The generation of identifiers and storing of information happen in real-time when the transactions occur. Therefore, the authorities and supply chain parties can easily monitor or track the transactions to protect the drug from counterfeiting and information from modification, thus establishing trust and reliability of the process. In addition, the process of recording and monitoring supply chain transactions electronically reduces cost and time and eliminates paperwork.

In the future, we want to implement our proposed model in other sectors, such as food and clothes, to provide reliability and traceability among vendors and customers. Furthermore, the Internet of Things IoT can be used to hold transaction information about physical things that are included in the ESC systems.

\section{ACKNOWLEDGMENT}

We would like to express thanks to Dr. Firdous Nuri, Dr. Dana Sardar, and Sulaimani Health Center for providing information about the drug manufacturer companies and pharmaceutical parties in Iraqi Kurdistan.

\section{REFERENCES}

[1] K. Korpela and T. Dahlberg. "Digital Supply Chain Transformation Toward Blockchain Integration". Hawaii International Conference on System Sciences (HICSS)At: Big Island, Hawaii, pp. 41824191, 2017.

[2] D. Schniederjans, C. Curado and M. Khalajhedayati. "Supply chain digitisation trends: An integration of knowledge management". International Journal of Production Economics, vol. 220, p. 107439, 2020. 
[3] S. Chen, R. Shi, Z. Ren, J. Yan, Y. Shi and J. Zhang. "A BlockchainBased Supply Chain Quality Management Framework". IEEE $14^{\text {th }}$ International Conference on e-Business Engineering (ICEBE), Shanghai, pp. 172-176, 2017.

[4] D. Tse, B. Zhang, Y. Yang, C. Cheng and H. Mu. "Blockchain Application in Food Supply Information Security". IEEE International Conference on Industrial Engineering and Engineering Management (IEEM), Singapore, pp. 1357-1361, 2017.

[5] W. Kenton. "Supply Chain". Available from: https://www. investopedia.com/terms/s/supplychain.asp. [Last accessed on 2020 Apr 01].

[6] N. B. Al Barghuthi, H. J. Mohamed and H. E. Said. "Blockchain in Supply Chain Trading". $5^{\text {th }}$ HCT Information Technology Trends (ITT), Dubai, United Arab Emirates, 2018, pp. 336-341, 2018.

[7] H. Min. "Blockchain technology for enhancing supply chain resilience". Business Horizons, vol. 62, no. 1, pp. 35-45, 2019.

[8] M. Nofer, P. Gomber, O. Hinz and D. Schiereck. "Blockchain". Business and Information Systems Engineering, vol. 59, no. 3, pp. 183-187, 2017.

[9] R. Kumar and R. Tripathi. "Traceability of Counterfeit Medicine Supply Chain through Blockchain". 11 $11^{\text {th }}$ International Conference on Communication Systems and Networks (COMSNETS), Bengaluru, India, pp. 568-570, 2019.

[10] S. Malik, S. S. Kanhere and R. Jurdak. "ProductChain: Scalable Blockchain Framework to Support Provenance in Supply Chains". IEEE $17^{\text {th }}$ International Symposium on Network Computing and Applications (NCA), Cambridge, MA, pp. 1-10, 2018.

[11] G. Büyüközkan and F. Göçer. "Digital supply chain: Literature review and a proposed framework for future research". Computers in Industry, vol. 97, pp. 157-177, 2018.

[12] S. Kamble, A. Gunasekaran and S. Gawankar. "Achieving sustainable performance in a data-driven agriculture supply chain: A review for research and applications". International Journal of Production Economics, vol. 219, pp. 179-194, 2020.

[13] S. Aich, S. Chakraborty, M. Sain, H. Lee and H. Kim. "A Review on Benefits of IoT Integrated Blockchain based Supply Chain Management Implementations across Different Sectors with Case Study". 21 $1^{\text {st }}$ International Conference on Advanced Communication Technology (ICACT), PyeongChang Kwangwoon Do, Korea
(South), pp. 138-141, 2019.

[14] S. R. Niya, D. Dordevic, A. G. Nabi, T. Mann and B. Stiller. "A Platform-independent, Generic-purpose, and Blockchain-based Supply Chain Tracking". IEEE International Conference on Blockchain and Cryptocurrency (ICBC), Seoul, Korea (South), pp. 11-12, 2019.

[15] V. Morkunas, J. Paschen and E. Boon. "How blockchain technologies impact your business model". Business Horizons, vol. 62, no. 3, pp. 295-306, 2019.

[16] C. Schmidt and S. Wagner. "Blockchain and supply chain relations: A transaction cost theory perspective". Journal of Purchasing and Supply Management, vol. 25, no. 4, p. 100552, 2019.

[17] D. Macrinici, C. Cartofeanu and S. Gao. "Smart contract applications within blockchain technology: A systematic mapping study". Telematics and Informatics, vol. 35, no. 8, pp. 2337-2354, 2018.

[18] S. S. Hazari and Q. H. Mahmoud. "A Parallel Proof of Work to Improve Transaction Speed and Scalability in Blockchain Systems". IEEE $9^{\text {th }}$ Annual Computing and Communication Workshop and Conference (CCWC), Las Vegas, NV, USA, pp. 916-921, 2019.

[19] A. A. Maksutov, M. S. Alexeev, N. O. Fedorova and D. A. Andreev, "Detection of Blockchain Transactions Used in Blockchain Mixer of Coin Join Type," 2019 IEEE Conference of Russian Young Researchers in Electrical and Electronic Engineering (EIConRus), Saint Petersburg and Moscow, Russia, pp. 274-277, 2019.

[20] S. Gueron. "Speeding Up SHA-1, SHA-256 and SHA-512 on the $2^{\text {nd }}$ Generation Inte/ ${ }^{\circledR}$ Core $^{\mathrm{TM}}$ Processors". 9 $9^{\text {th }}$ International Conference on Information Technology New Generations, Las Vegas, NV, pp. 824-826, 2012.

[21] A. Karakra and A. Alsadeh. "A-RSA: Augmented RSA". SAI Computing Conference (SAI), London, pp. 1016-1023, 2016.

[22] S. A. Nagar and S. Alshamma. "High Speed Implementation of RSA Algorithm with Modified Keys Exchange". 6 $6^{\text {th }}$ International Conference on Sciences of Electronics, Technologies of Information and Telecommunications (SETIT), Sousse, pp. 639-642, 2012.

[23] M. P. Caro, M. S. Ali, M. Vecchio and R. Giaffreda. "Blockchainbased Traceability in Agri-food Supply Chain Management: A Practical Implementation". IoT Vertical and Topical Summit on Agriculture Tuscany (IOT Tuscany), Tuscany, pp. 1-4, 2018. 